\title{
Analysing domain suitability of a sentiment lexicon by identifying distributionally bipolar words
}

\author{
Lucie Flekova $^{1 *}$, Eugen Ruppert ${ }^{2}$ and Daniel Preoţiuc-Pietro ${ }^{3}$ \\ ${ }^{1}$ Ubiquitous Knowledge Processing Lab, Technische Universität Darmstadt \\ ${ }^{2}$ FG Language Technology, Technische Universität Darmstadt \\ ${ }^{3}$ Computer \& Information Science, University of Pennsylvania \\ flekova@ukp.informatik.tu-darmstadt.de,ruppert@1t.tu-darmstadt.de,danielpr@sas.upenn.edu
}

\begin{abstract}
Contemporary sentiment analysis approaches rely heavily on lexicon based methods. This is mainly due to their simplicity, although the best empirical results can be achieved by more complex techniques. We introduce a method to assess suitability of generic sentiment lexicons for a given domain, namely to identify frequent bigrams where a polar word switches polarity. Our bigrams are scored using Lexicographers Mutual Information and leveraging large automatically obtained corpora. Our score matches human perception of polarity and demonstrates improvements in classification results using our enhanced contextaware method. Our method enhances the assessment of lexicon based sentiment detection algorithms and can be further used to quantify ambiguous words.
\end{abstract}

\section{Introduction}

Sentiment prediction from microblogging posts is of the utmost interest for researchers as well as commercial organizations. State-of-the-art sentiment research often focuses on in-depth semantic understanding of emotional constructs (Trivedi and Eisenstein, 2013; Cambria et al., 2013; De Marneffe et al., 2010) or neural network models (Socher et al., 2013; Severyn and Moschitti, 2015). However, recent sentiment prediction challenges show that the vast majority of currently used systems is still based on supervised learning techniques with the most important features derived from preexisting sentiment lexica (Rosenthal et al., 2014; Rosenthal et al., 2015).

Sentiment lexicons were initially developed as general-purpose resources (Pennebaker et al., 2001;

${ }^{*}$ Project carried out during a research stay at the University of Pennsylvania
Strapparava et al., 2004; Hu and Liu, 2004; Wilson et al., 2005). Recently, there has been an increasing amount of work on platform-specific lexicons such as Twitter (Mohammad, 2012; Mohammad et al., 2013). However, even customized platform- and domain-specific lexica still suffer from ambiguities at a contextual level, e.g. cold beer (+) or cold food $(-)$, dark chocolate (+) or dark soul (-).

In this paper, we propose a method to assess the suitability of an established lexicon for a new platform or domain by leveraging automatically collected data approximating sentiment labels (silver standard). We present a method for creating switched polarity bigram lists to explicitly reveal and address the issues of a lexicon in question (e.g. the positivity of cold beer, dark chocolate or limited edition). Note that the contextual polarity switch does not necessarily happen on sense level, but within one word sense. We demonstrate that the explicit usage of such inverse polarity bigrams and replacement of the words with high ambiguity improves the performance of the classifier on unseen test data and that this improvement exceeds the performance of simply using all in-domain bigrams. Further, our bigram ranking method is evaluated by human raters, showing high face validity.

\section{Related Work}

Sentiment research has tremendously expanded in the past decade. Overall, sentiment lexicons are the most popular inputs to polarity classification (Rosenthal et al., 2015; Rosenthal et al., 2014), although the lexicons alone are far from sufficient. Initial studies relied heavily on explicit, manually crafted sentiment lexicons (Kim and Hovy, 2004; Pang and Lee, 2004; Hu and Liu, 2004). There have been efforts to infer the polarity lexicons automatically. Turney and Littman (2003) determined the semantic orientation of a target word $t$ by comparing its association with two seed sets of manually crafted target words. Others derived the polar- 
ity from other lexicons (Baccianella et al., 2010; Mohammad et al., 2009), and adapted lexicons to specific domains, for example using integer linear programming (Choi and Cardie, 2009).

Lexicons are not stable across time and domain Cook and Stevenson (2010) proposed a method to compare dictionaries for amelioration and pejoration of words over time. Mitra et al. (2014) analyzed changes in senses over time. Dragut et al. (2012) examined inconsistency across lexicons.

Negation and its scope has been studied extensively (Moilanen and Pulman, 2008; Pang and Lee, 2004; Choi and Cardie, 2009). Polar words can even carry an opposite sentiment in a new domain (Blitzer et al., 2007; Andreevskaia and Bergler, 2006; Schwartz et al., 2013; Wilson et al., 2005). Wilson et al. (2005) identified polarity shifter words to adjust the sentiment on phrase level. Choi and Cardie (2009) validated that topic-specific features would enhance existing sentiment classifiers. Ikeda et al. (2008) first proposed a machine learning approach to detect polarity shifting for sentencelevel sentiment classification. Taboada et al. (2011) presented a polarity lexicon with negation words and intensifiers, which they refer to as contextual valence shifters (Polanyi and Zaenen, 2006). Research by Kennedy and Inkpen (2006) dealt with negation and intensity by creating a discrete modifier scale, namely, the occurrence of good might be either good, not good, intensified good, or diminished good. A similar approach was taken by Steinberger et al. (2012). Polarity modifiers, however, do not distinguish cases such as cannot be bad from cannot be worse.

Further experiments revealed that some nouns can carry sentiment per se (e.g. chocolate, injury). Recently, several noun connotation lexicons have been built (Feng et al., 2013; Klenner et al., 2014) based on a set of seed adjectives. One of the biggest disadvantages of polarity lexicons, however, is that they rely on either positive or negative score of a word, while in reality it can be used in both contexts even within the same domain (Volkova et al., 2013).

\section{Method}

This section describes our methodology for identifying ambiguous sentiment bearing lexicon words based on the contexts they appear in. We demonstrate our approach on two polarity lexicons consisting of single words, namely the lexicon of $\mathrm{Hu}$ and Liu (Hu and Liu, 2004), further denoted HL, and the MPQA lexicon (Wilson et al., 2005). First we use a corpus of automatically collected Twitter sentiment data set of over one million tweets (detailed in section 3.2) to compute bigram polarities for the lexicon words and determine contexts which alter the polarity of the original lexicon word. Using the JoBimText framework (Biemann and Riedl, 2013), we build a large Twitter bigram thesaurus which serves as a background frequency distribution which aids in ranking the bigrams (see section 3.1). For each lexicon word, we then replace the most ambiguous words with bigrams. We compare this on sentiment prediction with a straightforward usage of all bigrams.

\subsection{Twitter Bigram Thesaurus}

Methods based on word co-occurrence have a long tradition in NLP research, being used in tasks such as collocation extraction or sentiment analysis. Turney and Littman (2003) used polarity seeds to measure words which co-occur with positive/negative contexts. However, the PMI is known to be sensitive to low count words and bigrams, overemphasising them over high frequency words. To account for this, we express the mutual information of a word bigram by means of Lexicographer's Mutual Information (LMI). ${ }^{1}$ The LMI, introduced by Kilgarriff et al. (2004), offers an advantage to Pointwise Mutual Information (PMI), as the scores are multiplied by the bigram frequency, boosting more frequent combinations of word (w) and context (c).

$$
\begin{aligned}
& \operatorname{PMI}(w, c)=\log _{2}\left(\frac{f(w, c)}{f(w) \cdot f(c)}\right) \\
& \operatorname{LMI}(w, c)=\operatorname{PMI}(w, c) \cdot f(w, c)
\end{aligned}
$$

\subsection{Bigram Sentiment Scores}

We compute the LMI over a corpus of positive, respectively negative tweets, in order to obtain positive $\left(\mathrm{LMI}_{\text {pos }}\right)$ and negative $\left(\mathrm{LMI}_{n e g}\right)$ bigram scores. We combine the following freely available data, leading to a large corpus of positive and negative tweets:

- 1.6 million automatically labeled tweets from the Sentiment140 data set (Go et al., 2009), collected by searching for positive and negative emoticons;

\footnotetext{
${ }^{1}$ An online demo illustrating the score values and distributional term similarities in this Twitter space can be found at the LT website http://maggie.lt.informatik. tu-darmstadt.de/jobimviz/
} 
- 7,000 manually labeled tweets from University of Michigan; ${ }^{2}$

- 5,500 manually labeled tweets from Niek J. Sanders; ${ }^{3}$

- 2,000 manually labeled tweets from the STSGold data set (Saif et al., 2013).

We filtered out fully duplicate messages, as these appear to bring more noise than realistic frequency information. The resulting corpus contains 794,000 positive and 791,000 negative tweets. In pursuance of comparability between the positive and negative LMI scores, we weight the bigrams by their relative frequency in the respective data set, thus discounting rare or evenly distributed bigrams, as illustrated for negative score in:

$$
\begin{aligned}
\operatorname{LMI}_{\text {neg }_{R E L}}(w, c)= & \operatorname{LMI}_{\text {neg }}(w, c) \cdot \\
& \frac{f_{\text {neg }}(w, c)}{f_{\text {neg }}(w, c)+f_{\text {pos }}(w, c)}
\end{aligned}
$$

Since the LMI scores from a limited sized data set are not the most reliable, we further boost them by incorporating scores from a background corpus $\left(\mathrm{LMI}_{G L O B}\right)$ - described below. This approach emphasizes significant bigrams, even when their score in one polarity data set is low:

$$
\begin{aligned}
\operatorname{LMI}_{n e g_{G L O B}}(w, c)= & \operatorname{LMI}_{n e g_{R E L}}(w, c) . \\
& \operatorname{LMI}_{G L O B}(w, c)
\end{aligned}
$$

As background data we use a Twitter corpus of $1 \%$ of all tweets from the year 2013, obtained through the Twitter Spritzer API. We filtered this corpus with a language filter, ${ }^{4}$ resulting in 460 million English tweets.

For each bigram, we then compute its semantic orientation:

$$
\mathrm{LMI}_{S O}=\mathrm{LMI}_{\text {pos }_{G L O B}}-\mathrm{LMI}_{n e g_{G L O B}}
$$

These two large bigram lists, which at this point still contain all bigrams from the Twitter sentiment corpus, are then filtered by sentiment lexica, as we are only interested in bigrams with at least one word from the original sentiment lexicon (containing sigle words). We chose two sentiment polarity lexica for our experiments:

\footnotetext{
${ }^{2}$ http://inclass.kaggle.com/c/ si650winter11/data

${ }^{3}$ http://www.sananalytics.com/lab/ twitter-sentiment/

${ }^{4}$ https://github.com/shuyo/ language-detection
}

- the HL lexicon (Hu and Liu, 2004) having 4,782 negative and 2,004 positive words (e.g. happy, good, bad);

- the MPQA sentiment lexicon (Wilson et al., 2005), with 1,751 positive and 2,693 negative words. $^{5}$

The most interesting candidates for a novel bigram sentiment lexicon are:

- bigrams containing a word from a negative lexicon, which has a positive semantic orientation $\mathrm{LMI}_{S O}$, i.e. having higher global LMI in the positive data set than in the negative;

- bigrams containing a word from a positive lexicon with negative semantic orientation $\mathrm{LMI}_{S O}$

The top ranked bigrams, where local contextualization reverts the original lexicon score, are listed for both lexicons in Table 1. We can observe that the polarity shifting occurs in a broad range of situations, e.g. by using polar word as an intensity expression (super tired), by using polar word in names (desperate housewives, frank iero), by using multiword expressions, idioms and collocations (cloud computing, sincere condolences, light bulbs), but also by adding a polar nominal context to the adjective (cold beer/person, dark chocolate/thoughts, stress reliever/management, guilty pleasurelfeeling).

\subsection{Quantifying Polarity}

We have shown how to identify words which switch to the opposite polarity based on their word context. Our next goal is to identify words which occur in many contexts with both the original and the switched polarity and therefore are, without further disambiguation, harmful in either of the lexicons. With this aim we calculate a polarity score $\mathrm{POL}_{\text {word }}$ for each word $(w)$ in the polarity lexicon, using the number of its positive and negative contexts determined by their semantic orientation $\mathrm{LMI}_{S O}$ as previously computed:

$$
\operatorname{POL}(w)=p_{\text {pos }}(w)-p_{\text {neg }}(w)
$$

where we define $p_{\text {pos }}(w)$ and $p_{\text {neg }}(w)$, as the count of positive and negative bigrams respectively, of a

\footnotetext{
${ }^{5}$ This lexicon also contains neutral words, which might be interesting for some applications. Since the HL lexicon does not feature neutral words, we chose to omit those entries for comparable results. The words in MPQA are further distinguished as 'strong' or 'weak' by POS tag. Since we do not maintain POS information in our distributional LMI lists, we chose to utilize all indicators equally.
} 


\begin{tabular}{|c|c|c|c|}
\hline \multicolumn{4}{|c|}{ Negative to Positive } \\
\hline \multicolumn{2}{|c|}{ HL } & \multicolumn{2}{|c|}{ MPQA } \\
\hline Word & Context & Word & Context \\
\hline limit & why- & vice & -versa \\
\hline sneak & -peek & stress & -reliever \\
\hline impossible & mission- & down & calmed- \\
\hline lazy & -sunday & deep & -breath \\
\hline desperate & -housewives & long & -awaited \\
\hline cold & -beer & cloud & -computing \\
\hline guilty & -pleasure & dark & -haired \\
\hline belated & -birthday & bloody & -mary \\
\hline \multicolumn{4}{|c|}{ Positive to Negative } \\
\hline \multicolumn{2}{|c|}{ HL } & \multicolumn{2}{|r|}{ MPQA } \\
\hline Word & Context & Word & Context \\
\hline luck & good- & super & -duper \\
\hline wisdom & -tooth & happy & -camper \\
\hline well & oh- & just & -puked \\
\hline work & gotta- & heart & -breaker \\
\hline hot & -outside & gold & -digger \\
\hline better & feels- & light & -bulbs \\
\hline super & -tired & sincere & -condolences \\
\hline enough & -money & frank & -iero \\
\hline
\end{tabular}

Table 1: Bigrams with opposite LMI sentiment orientation than the original lexicon word. Note that the polarity rarely changes on sense level i.e., same sense can have different polar contexts.

lexicon word, divided by the count of all bigrams of that word:

$$
p_{\text {neg }}(w)=\frac{\sum(w, c)_{\forall(w, c): \operatorname{LMI}_{S O}<0}}{\sum(w, c)}
$$

Lexicon words with the lowest absolute polarity score and the highest number of different contexts $(\mathrm{w}, \mathrm{c})$ are listed in Table 2.

\section{Experiments}

To evaluate the quality of our bigrams, we perform two studies. First, we rate our inverted polarity bigrams intrinsically using crowdsourced annotations. Second, we assess the performance of the original and adjusted lexicons on a distinct expertconstructed data set of 1,600 Facebook messages annotated for sentiment. The disambiguated bigram lexicons are available on author's website.

\subsection{Intrinsic Evaluation}

We crowdsource ratings for the inverted polarity bigrams found using both the HL and MPQA lexicon. The raters were presented a list of 100 bigrams of each lexicon, with $25 \%$ having the same positive polarity as in the original lexicon, $25 \%$ the same negative polarity, $25 \%$ switching polarity from positive unigram to negative bigram and the remaining

\begin{tabular}{|c|c|c|c|c|}
\hline \multicolumn{5}{|c|}{ HL } \\
\hline Word & $\operatorname{POL}(w)$ & $\#(w, c)_{p o s}$ & $\#(w, c)_{n e g}$ & orig \\
\hline hot & .022 & 1151 & 1101 & + \\
\hline support & .022 & 517 & 494 & + \\
\hline important & -.023 & 204 & 214 & + \\
\hline super & -.043 & 734 & 801 & + \\
\hline crazy & -.045 & 809 & 886 & - \\
\hline right & -.065 & 3061 & 3491 & + \\
\hline proper & -.093 & 242 & 292 & + \\
\hline worked & -.111 & 275 & 344 & + \\
\hline top & .113 & 516 & 411 & + \\
\hline enough & -.114 & 927 & 1167 & + \\
\hline hell & .115 & 616 & 488 & - \\
\hline \multicolumn{5}{|c|}{ MPQA } \\
\hline Word & $\operatorname{POL}(w)$ & $\#(w, c)_{p o s}$ & $\#(w, c)_{n e g}$ & orig \\
\hline just & -.002 & 742 & 738 & + \\
\hline less & .009 & 51 & 50 & - \\
\hline sound & -.011 & 43 & 44 & + \\
\hline real & .027 & 35 & 37 & + \\
\hline little & .032 & 354 & 332 & - \\
\hline help & -.037 & 42 & 39 & + \\
\hline back & -.046 & 191 & 174 & + \\
\hline mean & .090 & 24 & 20 & - \\
\hline down & -.216 & 154 & 239 & - \\
\hline too & -.239 & 252 & 411 & - \\
\hline
\end{tabular}

Table 2: Most ambiguous sentiment lexicon words. Table displays the proportion of their positive and negative contexts and the original lexicon polarity.

quarter vice versa. They had to answer the question 'Which polarity does this word pair have?', given positive, negative and also neutral as options. Each bigram is rated by three annotators and the majority vote is selected. The inter-annotator agreement is measured using weighted Cohen's $\kappa$ (Cohen, 1968), which is especially useful for ordered annotations, as it accounts not only for chance, but also for the seriousness of a disagreement between annotators. $\kappa$ can range from -1 to 1 , where the value of 0 represents an agreement equal to chance while 1 equals to a perfect agreement, i.e. identical annotation values. We obtained an agreement of weighted Cohen's $\kappa=0.55$, which represents a "moderate agreement" (Landis and Koch, 1977). The confusion matrix of average human judgement compared to our computed bigram polarity is shown in Table 3. Some of the bigrams, especially for the MPQA lexicon, were assessed as objective, which our LMI method unfortunately does not reflect beyond the score value (neutral words are less polar). However, the confusion between negatively and positively labeled bigrams was very low. 


\begin{tabular}{lccc|ccc}
\hline & \multicolumn{3}{c}{ HL } & \multicolumn{3}{c}{ MPQA } \\
& Pos. & Neu. & Neg. & Pos. & Neu. & Neg. \\
\hline Pos. & 30 & 10 & 9 & 21 & 24 & 3 \\
Neg. & 11 & 10 & 30 & 5 & 18 & 25 \\
\hline
\end{tabular}

Table 3: Confusion matrix for the majority vote of word polarity by three annotators.

\subsection{Extrinsic Evaluation}

We evaluate our method on a data set of Facebook posts annotated for positive and negative sentiment by two psychologists. The posts are annotated on a scale from 1 to 9 , with 1 indicating strong negative sentiment and 9 strong positive sentiment. An average rating between annotators is considered to be the final message score. Ratings follow a normal distribution, i.e. with more messages having less polar score. An inter-annotator agreement of weighted Cohen's $\kappa=0.61$ on exact score was reached, representing a "substantial agreeement"(Landis and Koch, 1977). Given our task, in which we attempt to improve on misleading bipolar words, we removed the posts annotated as neutral (rating 5.0). This left us with 2,087 posts, of which we use only those containing at least one word from the polarity lexicons of our interest, i.e., 1,601 posts for MPQA and 1,526 posts for HL. We then compute a sentiment score of a post as a difference of positive and negative word counts present in the post. If a bigram containing the lexicon word is found, its $\mathrm{LMI}_{S O}$ score is used instead of the lexicon word polarity score. For the two lexicons and their modifications, we employ two evaluation measures Pearson correlation of the sentiment score of a post with the affect score, and classification accuracy on binary label, i.e., distinguishing if the affect is negative (1-4) or positive (6-9). Table 4 presents our results of four experiments using the following features:

- using the original unigram lexicon only (1);

- using original lexicon corrected by polarity score of lexicon bigrams when they appear (2-4);

- using pruned unigram lexicon, removing words that exceed entropy threshold of 0.99 or appear in more contexts of the opposite polarity than of the assumed one (5);

- using pruned unigram lexicon corrected by polarity score of (unpruned) lexicon bigrams when they appear (6-8);

- all bigrams (9).

\begin{tabular}{l|l|ll|ll}
\multicolumn{2}{l}{} & \multicolumn{2}{c}{ HL } & \multicolumn{2}{c}{ MPQA } \\
Id & Features & Acc. & Corr. & Acc. & Corr. \\
\hline 1 & Unigrams & .7070 & .5828 & .6608 & .4473 \\
2 & Unigrams + Bigrams & .7215 & .5959 & .6633 & .4478 \\
3 & Unigrams + Bigrams $_{+}$ & .7123 & .5928 & .6621 & .4468 \\
4 & Unigrams + Bigrams & .7163 & .5973 & .6621 & .4472 \\
5 & Pruned & .7228 & .6131 & .6627 & .4817 \\
6 & Pruned + Bigrams & .7333 & .5943 & .6646 & .4917 \\
7 & Pruned + Bigrams & .7150 & .6264 & .6633 & .4907 \\
8 & Pruned + Bigrams & .7287 & .6330 & .6640 & .4929 \\
9 & All in-domain Bigrams & .6907 & .1837 & $\mathbf{. 7 0 0 8}$ & .1812
\end{tabular}

Table 4: Predictive performance using lexicon based methods, displaying the classification accuracy and linear correlation of the affect score to LMI. Using McNemar's two-tailed test, there is a significant difference on $\mathrm{p}<0.05$ level between the runs 1 and 2, 5 and 6 and 1 and 5 for BL, and between the runs 1 and 6 for MPQA.

Table 4 shows that adding contextual bigrams brings a consistent improvement ( 1 vs. 2,5 vs. 6 ). Especially the negative part of the bigram lexica, including bigrams of negative words which have positive orientation, consistently improves results (1 vs. 4,5 vs. 8 ). Likewise, pruning of the lexicon with the polar entropy score ( 1 vs. 5) enhances the sentiment prediction performance. For both polarity lexicons the best performance is achieved by combining the two effects (8).

In case of the first lexicon, the performance is even higher than in case of applying for the same data a fully in-domain bigram lexicon, generated from the same large public Twitter corpus (Mohammad et al., 2013).

The correction of negative unigrams to positive bigrams does not improve the prediction as much as its counterpart. The main cause appears to be the fact that those expressions with shifted polarity shall be rather neutral - as discussed in section 4.1 and by some recent research (Zhu et al., 2014).

\subsection{Discussion}

Usage of bigrams does not always bring improvement, but sometimes also introduces new errors. One of the frequent sources of errors appears to be the remaining ambiguity of the bigrams due to more complex phrase structure. While the bigrams are tremendously helpful in message chunks such as 'holy shit, tech support...', where the holy $(+1)$ and support $(+1)$ is replaced by its appropriately polar contexts $(-0.35,-0.85)$, the same replacement is harmful in a post 'holy shit monday night was amazing'. Same applies for bigrams such as work ahead (-0.89) in 'new house....yeah!! lots of work ahead of us!!!' or nice outside (-0.65) in 'it's nice outside today!'. 
Additionally, the performance suffers when a longer negation window is applied, such as feeling sick in the post 'Isn't feeling sick woohoo!'. In our setup we did not employ explicit polarity switchers commonly used with word lexicons (Wilson et al., 2005; Pang and Lee, 2008; Steinberger et al., 2012) since the context captured by the bigrams often incorporated subtle negation hints per se, including their misspelled variations. This would make the combination of bigrams with more sophisticated syntactic features challenging.

Another very interesting issue are the bigrams which are explicitly positive but have learnt their negative connotation from a broader context, such as happy camper or looking good, which are more often used jointly with negations. Posts that use these bigrams without negation ('someone is a happy camper!') then lead to errors, and similarly a manual human assessment without a longer context fails. This issue concerns distributional approaches in general.

Lastly, several errors arise from the non-standard, slang and misspelled words which are not present often enough in our silver standard corpus. For example, while love you is clearly positive, love ya has a negative score. On corpora such as Twitter, further optimization of word frequency thresholds in lexical methods requires special attention.

\section{Conclusion}

Lexicon based methods currently remain, due to their simplicity, the most prevalent sentiment analysis approaches. While it is taken for granted that using more in-domain training data is always helpful, a little attention has been given to determining how much and why a given general-purpose lexicon can help in a specific target domain or platform. We introduced a method to identify frequent bigrams where a word switches polarity, and to find out which words are bipolar to the extent that it is better to have them removed from the polarity lexica. We demonstrated that our scores match human perception of polarity and bring improvement in the classification results using our enhanced context-aware method. Our method enhances the assessment of lexicon based sentiment detection algorithms and can be further used to quantify ambiguous words.

\section{Acknowledgements}

LF and DP-P acknowledge the support from Templeton Religion Trust, grant TRT-0048. The work of ER has been supported by the German Federal Ministry of Education and Research (BMBF) within the context of the Software Campus project LiCoRes under grant No. 01IS12054

\section{References}

Alina Andreevskaia and Sabine Bergler. 2006. Mining WordNet for a fuzzy sentiment: Sentiment tag extraction from WordNet glosses. In Proceedings of the 11th Conference of the European Chapter of the Association for Computational Linguistics, EACL, pages 209-216.

Stefano Baccianella, Andrea Esuli, and Fabrizio Sebastiani. 2010. SentiWordNet 3.0: An enhanced lexical resource for sentiment analysis and opinion mining. In Proceedings of the Seventh International Conference on Language Resources and Evaluation, LREC, pages 2200-2204.

Chris Biemann and Martin Riedl. 2013. Text: Now in 2D! a framework for lexical expansion with contextual similarity. Journal of Language Modelling, 1(1):55-95.

John Blitzer, Mark Dredze, and Fernando Pereira. 2007. Biographies, Bollywood, boom-boxes and blenders: Domain adaptation for sentiment classification. In Proceedings of the 45th Annual Meeting of the Association for Computational Linguistics, ACL, pages 440-447.

Erik Cambria, Bjorn Schuller, Yunqing Xia, and Catherine Havasi. 2013. New avenues in opinion mining and sentiment analysis. IEEE Intelligent Systems, (2):15-21.

Yejin Choi and Claire Cardie. 2009. Adapting a polarity lexicon using integer linear programming for domain-specific sentiment classification. In Proceedings of the 2009 Conference on Empirical Methods in Natural Language Processing, EMNLP, pages 590598.

Jacob Cohen. 1968. Weighted kappa: Nominal scale agreement provision for scaled disagreement or partial credit. Psychological bulletin, 70(4).

Paul Cook and Suzanne Stevenson. 2010. Automatically identifying changes in the semantic Ooientation of words. In Proceedings of the Seventh International Conference on Language Resources and Evaluation, LREC, pages 129-149.

Marie-Catherine De Marneffe, Christopher D Manning, and Christopher Potts. 2010. Was it good? It was provocative. Learning the meaning of scalar adjectives. In Proceedings of the 48th Annual Meeting of 
the Association for Computational Linguistics, ACL, pages 167-176.

Eduard Dragut, Hong Wang, Clement Yu, Prasad Sistla, and Weiyi Meng. 2012. Polarity consistency checking for sentiment dictionaries. In Proceedings of the 50th Annual Meeting of the Association for Computational Linguistics, ACL, pages 997-1005.

Song Feng, Jun Seok Kang, Polina Kuznetsova, and Yejin Choi. 2013. Connotation lexicon: A dash of sentiment beneath the surface meaning. In Proceedings of the 51st Annual Meeting of the Association for Computational Linguistics, ACL, pages 1774-1784.

Alec Go, Richa Bhayani, and Lei Huang. 2009. Twitter sentiment classification using distant supervision. CS224N Project Report, Stanford, 1.

Minqing $\mathrm{Hu}$ and Bing Liu. 2004. Mining and summarizing customer reviews. In Proceedings of the tenth ACM SIGKDD International conference on Knowledge Discovery and Data Mining, KDD, pages 168 177.

Daisuke Ikeda, Hiroya Takamura, Lev-Arie Ratinov, and Manabu Okumura. 2008. Learning to shift the polarity of words for sentiment classification. In Proceedings of the International Joint Conference on Natural Language Processing, IJCNLP, pages 296303.

Alistair Kennedy and Diana Inkpen. 2006. Sentiment classification of movie reviews using contextual valence shifters. Computational Intelligence, 22(2):110-125.

Adam Kilgarriff, Pavel Rychly, Pavel Smrz, and David Tugwell. 2004. Itri-04-08 the sketch engine. Information Technology, 105.

Soo-Min Kim and Eduard Hovy. 2004. Determining the sentiment of opinions. In Proceedings of the 20th international conference on Computational Linguistics, COLING, pages 1367-1378.

Manfred Klenner, Michael Amsler, and Nora Hollenstein. 2014. Inducing domain-specific noun polarity guided by domain-independent polarity preferences of adjectives. In Proceedings of the 52nd Annual Meeting of the Association for Computational Linguistics, ACL, pages 18-23.

J Richard Landis and Gary G Koch. 1977. The measurement of observer agreement for categorical data. biometrics, pages 159-174.

Sunny Mitra, Ritwik Mitra, Martin Riedl, Chris Biemann, Animesh Mukherjee, and Pawan Goyal. 2014 That's sick dude!: Automatic identification of word sense change across different timescales. In Proceedings of the 52nd Annual Meeting of the Association for Computational Linguistics, ACL, pages 1020-1029.
Saif Mohammad, Cody Dunne, and Bonnie Dorr. 2009. Generating high-coverage semantic orientation lexicons from overtly marked words and a thesaurus. In Proceedings of the 2009 Conference on Empirical Methods in Natural Language Processing, EMNLP, pages 599-608.

Saif Mohammad, Svetlana Kiritchenko, and Xiaodan Zhu. 2013. NRC-Canada: Building the state-of-theart in sentiment analysis of tweets. In Second Joint Conference on Lexical and Computational Semantics, volume 2 of $*$ SeM, pages 321-327.

Saif Mohammad. 2012. \#emotional tweets. In Proceedings of the First Joint Conference on Lexical and Computational Semantics, *SEM, pages 246-255.

Karo Moilanen and Stephen Pulman. 2008. The good, the bad, and the unknown: Morphosyllabic sentiment tagging of unseen words. In Proceedings of the 46th Annual Meeting of the Association for Computational Linguistics on Human Language Technologies, ACL, pages 109-112.

Bo Pang and Lillian Lee. 2004. A sentimental education: Sentiment analysis using subjectivity summarization based on minimum cuts. In Proceedings of the 42nd annual meeting on Association for Computational Linguistics, ACL, pages 271-278.

Bo Pang and Lillian Lee. 2008. Opinion mining and sentiment analysis. Foundations and Trends in Information Retrieval, 2:1-135.

James W. Pennebaker, Martha E. Francis, and Roger J. Booth. 2001. Linguistic Inquiry and Word Count.

Livia Polanyi and Annie Zaenen. 2006. Contextual valence shifters. In Computing attitude and affect in text: Theory and applications, pages 1-10. Springer.

Sara Rosenthal, Preslav Nakov, Alan Ritter, and Veselin Stoyanov. 2014. Semeval-2014 task 9: Sentiment analysis in Twitter.

Sara Rosenthal, Preslav Nakov, Svetlana Kiritchenko, Saif Mohammad, Alan Ritter, and Veselin Stoyanov. 2015. Semeval-2015 task 10: Sentiment analysis in Twitter. In Proceedings of the 9th International Workshop on Semantic Evaluation, SemEval.

Hassan Saif, Miriam Fernandez, Yulan He, and Harith Alani. 2013. Evaluation datasets for Twitter sentiment analysis: A survey and a new dataset, the STSgold. In Proceedings of the 1st International Workshop on Emotion and Sentiment in Social and Expressive Media: Approaches and Perspectives from AI, ESSEM.

H Andrew Schwartz, Johannes C Eichstaedt, Lukasz Dziurzynski, Margaret L Kern, Eduardo Blanco, Stephanie Ramones, Martin E P Seligman, and Lyle H Ungar. 2013. Choosing the right words: Characterizing and reducing error of the word count approach. In Proceedings of the Second Joint Conference on Lexical and Computational Semantics, *SEM, pages 296-305. 
Aliaksei Severyn and Alessandro Moschitti. 2015. Twitter sentiment analysis with deep convolutional neural networks. In Proceedings of the 38th International ACM SIGIR Conference on Research and Development in Information Retrieval, pages 959-962.

Richard Socher, Alex Perelygin, Jean Y Wu, Jason Chuang, Christopher D Manning, Andrew Y Ng, and Christopher Potts. 2013. Recursive deep models for semantic compositionality over a sentiment treebank. In Proceedings of the 2012 Joint Conference on Empirical Methods in Natural Language Processing and Computational Natural Language Learning, EMNLP, pages 1642-1649.

Josef Steinberger, Mohamed Ebrahim, Maud Ehrmann, Ali Hurriyetoglu, Mijail Kabadjov, Polina Lenkova, Ralf Steinberger, Hristo Tanev, Silvia Vazquez, and Vanni Zavarella. 2012. Creating sentiment dictionaries via triangulation. Decision Support Systems, 53(4).

Carlo Strapparava, Alessandro Valitutti, et al. 2004. WordNet Affect: An affective extension of WordNet. In Proceedings of the Fourth International Conference on Language Resources and Evaluation, LREC, pages 1083-1086.

Maite Taboada, Julian Brooke, Milan Tofiloski, Kimberly Voll, and Manfred Stede. 2011. Lexicon-based methods for sentiment analysis. Computational Linguistics, 37(2):267-307.

Rakshit S Trivedi and Jacob Eisenstein. 2013. Discourse connectors for latent subjectivity in sentiment analysis. In HLT-NAACL, pages 808-813.

Peter D Turney and Michael L Littman. 2003. Measuring praise and criticism: Inference of semantic orientation from association. ACM Transactions on Information Systems, 21(4):315-346.

Svitlana Volkova, Theresa Wilson, and David Yarowsky. 2013. Exploring sentiment in social media: Bootstrapping subjectivity clues from multilingual twitter streams. In Proceedings of the 51st Annual Meeting of the Association for Computational Linguistics on Human Language Technologies, ACL, pages 505-510.

Theresa Wilson, Janyce Wiebe, and Paul Hoffmann. 2005. Recognizing contextual polarity in phraselevel sentiment analysis. In Proceedings of the 2005 Joint Conference on Empirical Methods in Natural Language Processing and Computational Natural Language Learning, EMNLP, pages 347-354.

Xiaodan Zhu, Hongyu Guo, Saif Mohammad, and Svetlana Kiritchenko. 2014. An empirical study on the effect of negation words on sentiment. In Proceedings of the 52rd Annual Meeting of the Association for Computational Linguistics, ACL, pages 304-313. 